\section{Opposing Associations of NT-proBNP With Risks of Diabetes and Diabetes-Related Complications}

Diabetes Care 2020;43:2930-2937 | https://doi.org/10.2337/dc20-0553

\section{OBJECTIVE}

Circulating N-terminal pro B-type natriuretic peptide (NT-proBNP) is a classic diagnostic and prognostic marker for heart failure. However, it is inversely associated with diabetes risk. We aimed to investigate relationships of NT-proBNP with risk of diabetes-related complications in initially healthy individuals.

\section{RESEARCH DESIGN AND METHODS}

We performed a case-cohort study within the European Prospective Investigation Into Cancer and Nutrition (EPIC)-Potsdam cohort including a random subcohort ( $\boldsymbol{n}=$ $1,294)$ and incident cases of type 2 diabetes $(n=649)$ and cardiovascular diseases ( $n=478$ ). Incident cases of type 2 diabetes $(n=545)$ were followed up for microvascular $(n=133)$ and macrovascular $(n=50)$ complications. Plasma NTproBNP was measured at baseline in initially healthy participants.

\section{RESULTS}

In multivariable models, NT-proBNP was linearly inversely associated with incident type 2 diabetes with a hazard ratio (HR) $(95 \% \mathrm{CI})$ per doubling in NT-proBNP of 0.91 $(0.86,0.98)$. The association was only observable in women $(0.80[0.72,0.90])$ compared with men $(0.98[0.91,1.07])$. Among people with incident diabetes, NT-proBNP was positively associated with diabetes complications: overall, 1.31 (1.13, 1.53); microvascular complications, 1.20 (1.01, 1.43); and macrovascular complications, 1.37 (1.03, 1.83).

\section{CONCLUSIONS}

Although higher NT-proBNP levels are associated with lower diabetes risk, NTproBNP is a biomarker for vascular complications in people who develop diabetes independent of potential confounders. Thus, NT-proBNP might be informative to monitor risk for diabetes-related microvascular and macrovascular complications, which should be further explored in future prospective studies.

Circulating N-terminal pro B-type natriuretic peptide (NT-proBNP) is an inactive amino-acid fragment of the brain natriuretic peptide (BNP) that is released from cardiomyocytes in response to volume or pressure overload, promoting vasodilation and natriuresis. As such, it is a classic marker of acute heart failure (HF) and left ventricular dysfunction. Several studies (1) have investigated relationships of NTproBNP with HF risk. However, NT-proBNP together with its receptors not only orchestrates cardiovascular homeostasis and health by regulating blood pressure,
Anna Birukov, ${ }^{1,2}$ Fabian Eichelmann, ${ }^{1,2}$ Olga Kuxhaus, ${ }^{1}$ Elli Polemiti, ${ }^{1,2}$ Andreas Fritsche, ${ }^{3}$ Janine Wirth, ${ }^{4}$ Heiner Boeing, ${ }^{5}$ Cornelia Weikert, ${ }^{6}$ and Matthias B. Schulze $e^{1,2,7}$
${ }^{1}$ Department of Molecular Epidemiology, German Institute of Human Nutrition PotsdamRehbrücke, Nuthetal, Germany

${ }^{2}$ German Center for Diabetes Research, MünchenNeuherberg, Germany

${ }^{3}$ Division of Endocrinology, Diabetology, Nephrology, Vascular Disease and Clinical Chemistry, Department of Internal Medicine, University of Tübingen, Tübingen, Germany

${ }^{4}$ Institute of Food and Health, School of Agriculture and Food Science, University College Dublin, Belfield, Dublin, Ireland

${ }^{5}$ Department of Epidemiology, German Institute of Human Nutrition Potsdam-Rehbrücke, Nuthetal, Germany

${ }^{6}$ Department of Food Safety, German Federal Institute for Risk Assessment, Berlin, Germany

${ }^{7}$ Germany Institute of Nutrition Science, University of Potsdam, Nuthetal, Germany

Corresponding author: Matthias B. Schulze, mschulze@dife.de

Received 18 March 2020 and accepted 23 July 2020

This article contains supplementary material online at https://doi.org/10.2337/figshare.12735440.

(C) 2020 by the American Diabetes Association. Readers may use this article as long as the work is properly cited, the use is educational and not for profit, and the work is not altered. More information is available at $h t t p s: / / w w w . d i a b e t e s j o u r n a l s$ .org/content/license.

See accompanying article, p. 2904. 
blood volume, and sodium balance but also it is involved in glucose and lipid metabolism in adipose and muscle tissues $(2,3)$. Natriuretic peptides stimulate lipolysis in adipocytes by binding to natriuretic peptide receptor A (NPR-A) in adipose tissue; promote browning of white adipocytes, oxygen consumption, and glucose uptake in white and brown adipocytes; regulate body fat distribution; and modulate food intake and energy expenditure $(2,3)$. Natriuretic peptides enhance adiponectin secretion from adipocytes via NPR-A, suggesting that NPR-A activation may modulate adipokine secretion and have a beneficial effect on low-grade inflammation of the adipose tissue and, thus, insulin resistance (4). Taken together, these biological effects of NT-proBNP benefit insulin and glucose metabolism. Prospective human cohort studies, including 2 Mendelian randomization studies $(5,6)$, have already reported an inverse association between baseline NT-proBNP levels and type 2 diabetes risk (5-8).

However, there is a lack of insight whether NT-proBNP levels relate to the risk of vascular complications of diabetes. Previous studies have been largely crosssectional in nature $(9,10)$ or investigated the risk of cardiovascular diseases (CVD) and cardiovascular mortality in populations with diabetes (11-15). There are well-recognized sex-specific differences in circulating NT-proBNP concentrations, with women showing higher NT-proBNP levels than men (16-18). We thus aimed to investigate the prospective associations of prediagnosis NT-proBNP levels with risk of microvascular and macrovascular complications in incident type 2 diabetes cases from a nested case-cohort consisting of initially healthy participants.

\section{RESEARCH DESIGN AND METHODS}

Study Design and Population

Our study is embedded in the European Prospective Investigation Into Cancer and Nutrition (EPIC)-Potsdam cohort, consisting of 27,548 individuals $(16,644$ women aged 35-65 years and 10,904 men aged 40-65 years) with available blood sample measurements (19). EPICPotsdam is a cohort study aiming to prospectively investigate the role of diet in the development of cancer and other chronic diseases (20). Participants were recruited between 1994 and 1998 from the general population of the Potsdam area in Germany. The study was conducted according to the Declaration of Helsinki and approved by the Ethical Committee of the State of Brandenburg, Germany. All participants provided written informed consent. At the time of recruitment, anthropometric and blood pressure measurements were taken, followed by an interview and a questionnaire on prevalent medical conditions, sociodemographic and lifestyle characteristics. Follow-up on incident diseases, diet, and other lifestyle factors was conducted every 2-3 years. Response rates ranged between 90 and $96 \%$ per followup round (21).

Analyses on incident type 2 diabetes and CVD were performed in a case-cohort setting. The nested case-cohort consisted of a random sample of EPIC-Potsdam participants who provided blood ( $n=$ $1,294)$ and all incident cases of type 2 diabetes $(n=820)$ or CVD $(n=518)$. Of these, 74 diabetes cases and 29 CVD cases were also part of the subcohort (internal cases). End of the follow-up was 31 August 2005 for diabetes and 30 November 2006 for CVD. After exclusion of participants with prevalent cardiometabolic conditions, missing follow-up time, or insufficient plasma samples, data on 1,656 and 1,648 participants were available for analyses on diabetes and CVD, respectively (Supplementary Figs. 1 and 2).

For the analyses on diabetes complications, all incident type 2 diabetes cases with available plasma samples from baseline and follow-up information on complications status formed the analytical sample ( $n=545$ ) (Supplementary Fig. 1).

\section{Case Ascertainment}

Systematic information sources for incident diabetes and CVD were self-report of a respective diagnosis, pharmacological treatment, or dietary change due to disease, collected through follow-up questionnaires every 2-3 years. Further sources of information included death certificates, tumor centers, and clinical records linkage. Verification of potential cases was carried out through standardized forms filled by the participants' treating physicians (20). Only physician-verified cases with a diagnosis date after the baseline examination were considered confirmed incident cases of type 2 diabetes or CVD (myocardial infarction [MI] or stroke). For diabetes- related complications, standardized forms were sent to the treating physicians of all incident diabetes cases in 2014, assessing the diagnosis of microvascular and macrovascular complications. Complications were considered incident if diagnosed after diabetes onset. The following disease outcomes according to the ICD-10 were investigated in this study: type 2 diabetes (ICD-10 E11) and CVD, comprising $\mathrm{MI}$ (ICD-10 I21) and stroke (ischemic, ICD-10 I63.0-163.9; hemorrhagic, ICD-10 160.0-161.9; and undetermined stroke, ICD-10 I64.0-I64.9). Microvascular complications comprised diabetic kidney disease (ICD-10 E11.2, including unspecified diabetes-related nephropathy and renal replacement therapy), retinopathy (ICD$10 \mathrm{E} 11.3$, including proliferative or nonproliferative retinopathy or blindness) and neuropathy (ICD-10 E11.4, including unspecified diabetes-related peripheral neuropathy or amputation due to diabetic neuropathy). Macrovascular complications encompassed $\mathrm{Ml}$ or stroke after diabetes diagnosis.

\section{Laboratory Analyses}

A sample of $30 \mathrm{~mL}$ blood was drawn from mainly nonfasting participants at baseline and collected in monovettes containing $10 \%$ citrate. Samples were aliquoted and stored in tanks of liquid nitrogen $\left(-196^{\circ} \mathrm{C}\right)$ or deep freezers $\left(-80^{\circ} \mathrm{C}\right)(20)$. Plasma NT-proBNP, estradiol, testosterone, and sex hormone-binding globulin (SHBG) concentrations were measured using a solid-phase two-site chemiluminescent immunometric assay (IMMULITE 2000 Systems Analyzers; SIEMENS, Eschborn, Germany) at the Institute of Clinical Chemistry, University of Magdeburg, Magdeburg, Germany. The assay has an effective measuring range of 20$35,000 \mathrm{pg} / \mathrm{mL}$. The median within-run coefficient of variation was $5.4 \%$ at a concentration of $35.6 \mathrm{pg} / \mathrm{mL}$ and $4.1 \%$ at a concentration of $29,725 \mathrm{pg} / \mathrm{mL}$. The overall coefficients of variation throughout the analyses were $6.4 \%$ and $4.7 \%$ at the same sample concentrations (18). Plasma adiponectin was measured with a sandwich ELISA (LINCO Research, St. Charles, MO); HDL and total cholesterol, triglycerides, hemoglobin $\mathrm{A}_{1 \mathrm{c}}\left(\mathrm{HbA}_{1 \mathrm{c}}\right)$, and hs-CRP were measured using an automatic ADVIA 1650 analyzer (Siemens Medical Solutions) at the University of Tübingen, Tübingen, Germany. All plasma biomarker measurements were corrected 
for the dilution introduced by citrate volume to improve comparability with concentrations measured in EDTA plasma (22).

\section{Statistical Analyses}

Baseline characteristics of the study participants were evaluated according to the quartiles of baseline NT-proBNP concentrations in the subcohort. Missing data were handled with multiple imputation, by applying the multivariate model with a fully conditional specification method; five imputations were conducted. In 324 (19.6\%) and 283 (17.2\%) participants of the case-cohorts for type 2 diabetes and CVD, the NT-proBNP concentration was below the limit of detection $(<20 \mathrm{pg}$ / $\mathrm{mL})$; thus, these values were imputed with multiple imputation using the uniform distribution method (23). The same method was applied for imputing testosterone and estradiol concentrations below limit of detection $(<0.69 \mathrm{nmol} / \mathrm{L}$ and $<73.4 \mathrm{pmol} / \mathrm{L}$, respectively). Apart from missing data due to it being below the limit of detection, missing values ranged between 0.4 and $3.6 \%$. Concentrations of NT-proBNP, triglycerides, hs-CRP, creatinine, testosterone, estradiol, SHBG, and adiponectin were nonnormally distributed; thus, $\log _{2}$-transformed values of these biomarkers were used in all analyses. Correlation between NT-proBNP and cardiometabolic risk factors (BMI, smoking, prevalent hypertension or hyperlipidemia, blood lipids, sex hormones, and other cardiovascular biomarkers) was assessed with age-adjusted Spearman correlation coefficients.

For associations between baseline NTproBNP concentrations and risk of type 2 diabetes and CVD, Prentice-weighted Cox models were used to account for the case-cohort design, stratified by age at recruitment. Age at recruitment was used as an underlying time scale. Followup in the case-cohort was defined as the time between enrollment and study exit (diagnosis of type 2 diabetes or CVD, death, drop out, or final censoring date, which ever came first). The basic multivariable model (model 1 ) was adjusted for sex, BMI $\left(\mathrm{kg} / \mathrm{m}^{2}\right)$, educational attainment (in training/or no certificate, professional school, or university degree), smoking status (current smoking: yes/no), alcohol intake (categories: <6.0, 6.1-12.0, 12.124.0, 24.1-60.0, 60.1-96.0 and >96.0 g/ day), recreational physical activity (average weekly hours), blood pressure, antihypertensive treatment, and prevalent conditions (hyperlipidemia; and for analyses on CVD risk: type 2 diabetes). The main model (model 2) was additionally adjusted for hs-CRP, total and HDL-cholesterol, triglycerides, estimated glomerular filtration rate (eGFR), and sex hormones (testosterone, estradiol, and SHBG). eGFR was estimated with the Chronic Kidney Disease Epidemiology Collaboration formula (24). The final model (model 3) was further adjusted for $\mathrm{HbA}_{1 \mathrm{c}}$ and adiponectin.

For associations between baseline NTproBNP concentrations and microvascular and macrovascular complications in people with incident diabetes, conventional Cox regression models, stratified by age at diabetes diagnosis, were applied. The covariate structure was the same as in the analyses on type 2 diabetes and CVD, except that models were additionally adjusted for the time interval between recruitment and diabetes diagnosis. Further, subjects' age at diabetes diagnosis was used as underlying time scale. Follow-up time in these analyses was defined as the time between diabetes diagnosis and diagnosis of the respective vascular complication, death, or date of last examination by the treating physician. The shape of the association with all outcomes was evaluated with restricted cubic spline regressions with three knots at 5th, 50th, and 95th percentile, applied on the fully adjusted model 2. The median $\log _{2}$-transformed NT-proBNP was used a reference. The nonlinear trend was assessed with the Wald test. We excluded individuals with baseline $\mathrm{HbA}_{1 \mathrm{c}} \geq 6.5 \%$ ( $n=249$ ) in the sensitivity analyses on type 2 diabetes and diabetes complications.

Finally, we tested whether the addition of NT-proBNP would improve the predictive performance of prediction models for type 2 diabetes and complications using c-index. For the prediction of diabetes, we used the German Diabetes Risk Score $(25,26)$. For the prediction of complications, we constructed a model comprising age, BMI, smoking status, systolic blood pressure, HDLcholesterol, triglycerides, eGFR, and $\mathrm{HbA}_{1 \mathrm{c}}$ based on the recent meta-analysis of 20 studies on the prediction of diabetic kidney disease (27).

The interaction between NT-proBNP and sex was tested by creating cross-product terms and evaluating the significance level.
A two-sided $P<0.05$ denoted statistical significance. All statistical analyses were performed using SAS (version 9.4, Enterprise Guide 7.1; SAS Institute Inc., Cary, NC).

\section{RESULTS}

\section{Characteristics of Study Participants} According to Baseline NT-proBNP

\section{Concentrations}

Median (interquartile range) follow-up times for type 2 diabetes, CVD, and diabetes complications (from diabetes diagnosis on) were 6.1 (3.5), 8.0 (2.5), and 12.5 (5.0) years, respectively.

Clinical and demographic characteristics of participants of the subcohort according to the distribution of baseline NT-proBNP are presented in Table 1. Study participants with higher NT-proBNP concentrations were on average older, more likely to be women, and had lower $\mathrm{BMI}$ and daily alcohol consumption. The fraction of hypertensive individuals increased across NT-proBNP quartiles. Concentrations of HDL-cholesterol, hs-CRP, estradiol, SHBG, and adiponectin increased on average and concentrations of triglycerides, creatinine, and testosterone decreased across NT-proBNP quartiles. NT-proBNP concentrations were weakly positively correlated to adiponectin ( $r=$ 0.18 in men and $r=0.08$ in women) and SHBG $(r=0.15$ in men and $r=0.21$ in women) and were inversely correlated to triglyceride concentrations ( $r=-0.16$ in men and $r=-0.04$ in women) and BMI $(r=-0.10$ in men and $r=-0.08$ in women) after adjustment for age (Supplementary Table 1).

NT-proBNP and Risk of Type 2 Diabetes There was an inverse association between NT-proBNP concentrations and future risk of type 2 diabetes (model 2, Fig. $1 A$, and Supplementary Table 2): each twofold increase in NT-proBNP was associated with $9 \%$ reduction in diabetes risk (hazard ratio [HR] 0.91; $95 \% \mathrm{Cl}$ $0.86,0.98)$. When tested for interaction in the basic model adjusted for lifestyle factors and prevalent conditions, we found a significant interaction between NT-proBNP and sex $(P=0.04)$. We thus stratified the analyses by sex and found a more pronounced association in women (model 2, Fig. 1B, and Supplementary Table 2): $20 \%$ reduction in type 2 diabetes risk with each doubling of NT-proBNP levels (HR 0.80; 95\% Cl 0.72, 0.90). In 
Table 1-Participants' characteristics of the subcohort according to quartiles of NT-proBNP, $n=1,064$

\begin{tabular}{|c|c|c|c|c|}
\hline & First quartile & Second quartile & Third quartile & Fourth quartile \\
\hline NT-proBNP, pg/mL & $18.4(13.2)$ & $40.8(11.4)$ & $65.8(17.2)$ & $130.2(72.5)$ \\
\hline \multicolumn{5}{|l|}{ Sociodemographics } \\
\hline Age at recruitment, years & $45.0(12.0)$ & $47.0(14.0)$ & $50.0(16.0)$ & $54.0(15.0)$ \\
\hline Age at type 2 diabetes diagnosis, years & $52.5(13.2)$ & $54.6(14.8)$ & $58.2(15.9)$ & 60.5 (14.9) \\
\hline Sex, women, $n(\%)$ & $91(34.0)$ & $159(60.2)$ & $206(76.6)$ & $203(77.2)$ \\
\hline $\mathrm{BMI}, \mathrm{kg} / \mathrm{m}^{2}$ & $26.2(4.7)$ & $25.0(5.4)$ & $24.6(4.9)$ & $25.1(5.6)$ \\
\hline Obesity, $n(\%)^{*}$ & $48(17.9)$ & $39(14.8)$ & $35(13.0)$ & $38(14.5)$ \\
\hline Higher education (university), $n(\%)$ & $108(40.3)$ & $101(38.3)$ & $96(35.7)$ & $92(35.0)$ \\
\hline Physical activity, h/week & $1.5(0.3)$ & $1.5(0.3)$ & $1.5(0.3)$ & $1.5(0.3)$ \\
\hline Alcohol consumption, g/day & $11.8(23.1)$ & $8.4(18.0)$ & $6.7(13.8)$ & $7.5(13.3)$ \\
\hline Smoking, $n(\%)$ & $61(22.8)$ & $64(24.2)$ & $54(20.1)$ & $43(16.4)$ \\
\hline eGFR, $\mathrm{mL} / \mathrm{min} / 1.73 \mathrm{~m}^{2}+$ & $95.5(20.0)$ & $95.1(22.6)$ & $91.4(24.3)$ & $90.3(21.2)$ \\
\hline $\mathrm{SBP}, \mathrm{mmHg}$ & $128.5(20.5)$ & $126.0(19.8)$ & $124.0(25.0)$ & $130.0(27.5)$ \\
\hline $\mathrm{DBP}, \mathrm{mmHg}$ & $83.5(13.0)$ & $81.5(16.0)$ & $81.0(14.0)$ & $83.0(16.5)$ \\
\hline Prevalent hypertension, $n(\%) \ddagger$ & $122(45.5)$ & $117(44.3)$ & $122(45.4)$ & $147(55.9)$ \\
\hline Prevalent hyperlipidemia, $n(\%)$ & $70(26.1)$ & $62(23.5)$ & $66(24.5)$ & $76(28.9)$ \\
\hline \multicolumn{5}{|l|}{ Biomarkers } \\
\hline $\mathrm{HbA}_{1 \mathrm{c}}, \%$ & $5.4(0.6)$ & $5.4(0.6)$ & $5.4(0.6)$ & $5.4(0.6)$ \\
\hline $\mathrm{HbA}_{1 \mathrm{c}}, \mathrm{mmol} / \mathrm{mol}$ & $36(6.6)$ & $36(6.6)$ & $36(6.6)$ & $36(6.6)$ \\
\hline Total cholesterol, $\mathrm{mg} / \mathrm{dL}$ & $206.6(57.5)$ & $200.0(50.0)$ & 205.8 (45.9) & $203.4(61.1)$ \\
\hline Triglycerides, $\mathrm{mg} / \mathrm{dL}$ & $126.8(108.4)$ & $97.4(91.5)$ & $102.3(72.1)$ & $101.1(71.1)$ \\
\hline HDL cholesterol, mg/dL & $49.6(17.6)$ & $56.2(18.6)$ & $57.1(20.3)$ & $57.7(19.5)$ \\
\hline hs-CRP, mg/dL & $0.06(0.2)$ & $0.06(0.2)$ & $0.07(0.2)$ & $0.10(0.2)$ \\
\hline Creatinine, $\mathrm{mg} / \mathrm{dL}$ & $0.9(0.2)$ & $0.8(0.2)$ & $0.8(0.2)$ & $0.8(0.2)$ \\
\hline Testosterone, $\mathrm{nmol} / \mathrm{L}$ & $9.4(13.0)$ & $1.1(10.6)$ & $0.7(1.1)$ & $0.7(1.1)$ \\
\hline SHBG, nmol/L & $35.3(23.8)$ & $50.8(39.7)$ & $54.1(41.0)$ & $59.8(48.8)$ \\
\hline Estradiol, pmol/L & $139.7(100.0)$ & 144.2 (173.1) & 155.8 (166.9) & 149.1 (158.5) \\
\hline Adiponectin, $\mu \mathrm{g} / \mathrm{mL}$ & $5.8(4.2)$ & $7.8(5.1)$ & $8.5(5.2)$ & $8.9(6.1)$ \\
\hline
\end{tabular}

Data are presented as median (interquartile range), unless otherwise noted. *Obesity was defined as $\mathrm{BMI} \geq 30 \mathrm{~kg} / \mathrm{m}^{2}$. teGFR estimated with Chronic Kidney Disease Epidemiology Collaboration formula. ‡Hypertension was defined as self-reported hypertension, or use of antihypertensive medication, or systolic blood pressure (SBP) $\geq 140 \mathrm{mmHg}$, or diastolic blood pressure (DBP) $\geq 90 \mathrm{mmHg}$ during blood pressure measurement in the study center at baseline examination.

men, the direction of the association was similar; however, Cox models showed no association (0.98; 0.91, 1.07) (model 2, Fig. $1 C$, and Supplementary Table 2). When adjusted for $\mathrm{HbA}_{1 \mathrm{c}}$ and adiponectin (model 3 ), the inverse association between NT-proBNP and risk of type 2 diabetes was slightly attenuated; however, it remained significant in women (Supplementary Table 2). Reduction in diabetes risk in women was observed independent of menopausal status $(P=$ 0.54 for interaction) (Supplementary Table 2). When we excluded participants with baseline $\mathrm{HbA}_{1 \mathrm{c}} \geq 6.5 \%$, the associations between NT-proBNP and type 2 diabetes risk were not substantially altered (data not shown). The results obtained by multiple imputation for NT-proBNP and steroid hormones were also well in agreement with estimates obtained by imputing the lowest detectable value (Supplementary Table 3).

\section{NT-proBNP and Risk of CVD}

No statistically significant association between circulating NT-proBNP and the risk of future CVD, MI, or stroke could be detected in any of the adjusted Cox models, regardless of sex of the participants (Supplementary Table 4). Inspection of the restricted cubic splines revealed a U-shaped relationship with $\mathrm{MI}$ risk in women ( $P=0.02$ ) (Supplementary Fig. 3B). No further violation of linearity of the associations could be seen in Supplementary Fig. $3 A$ and $C$.

\section{NT-proBNP and Risk of Microvascular} and Macrovascular Complications

Next, we investigated the relationships of NT-proBNP, measured at recruitment in healthy individuals, with subsequent risk of diabetes-related vascular complications. Participants destined to develop vascular complications of diabetes were on average older, more likely to be men, and had a more unfavorable cardiometabolic profile characterized by higher triglycerides, lower HDL-cholesterol, lower adiponectin, and higher NT-proBNP concentrations (Supplementary Table 5). Density distribution of NT-proBNP concentrations stratified by diabetes complications status and sex revealed different distribution patterns between men and women (Fig. 2). On average, women had higher NT-proBNP concentrations than men. The distribution of NT-proBNP in women who later developed type 2 diabetes without complications was shifted to lower concentrations as compared with their counterparts with vascular complications (Fig. $2 B)$, but no such trend was apparent in the male population (Fig. $2 C$ ).

In multivariable Cox regression analyses, higher baseline NT-proBNP concentrations were linearly positively associated with higher risk of microvascular and macrovascular complications (Fig. 3 and Supplementary Table 6). Each doubling in NT-proBNP concentration was associated with a 1.31-times higher risk of any diabetes complications $(95 \% \mathrm{Cl} 1.13$, 1.53) (Fig. $3 A$ and Supplementary Table 6), a 1.20-times higher risk of microvascular complications (1.01, 1.43) (Fig. $3 B$ and Supplementary Table 6), and a 1.37-times higher risk of macrovascular complications $(1.03,1.83$ ) (Fig. 3C and Supplementary Table 6). The associations for 

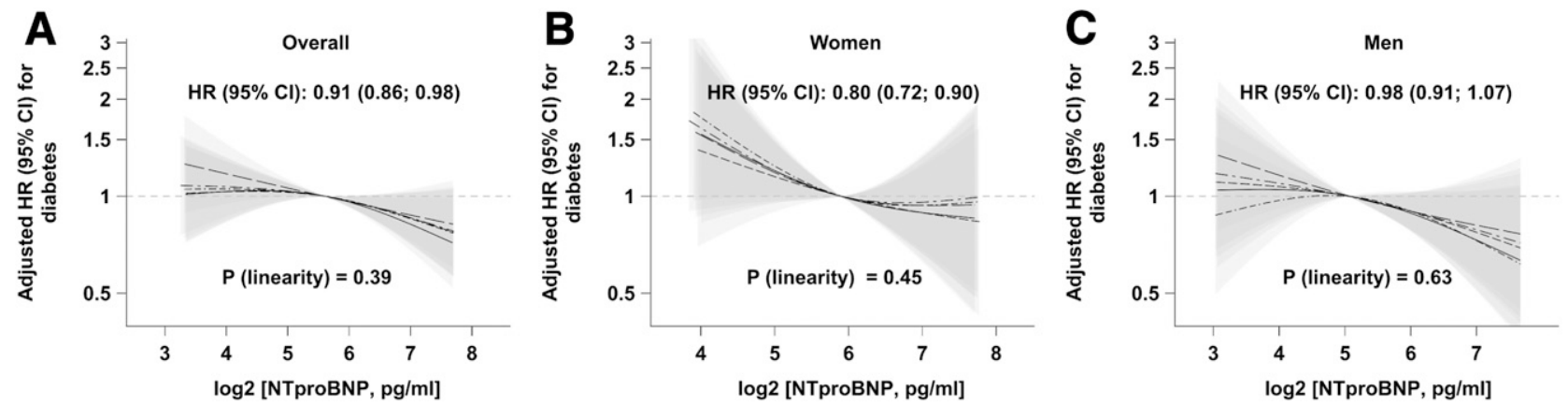

Figure 1-Sex-specific associations of baseline NT-proBNP concentrations with risk of type 2 diabetes in the case-cohort of the EPIC-Potsdam study $(n=$ 1,656). Adjusted HRs per doubling in NT-proBNP concentrations were assessed with Cox proportional hazards model (main model 2 ) and are shown with corresponding $95 \% \mathrm{Cl}$ in the middle of each panel. Linearity of the relationship between NT-proBNP concentrations and risk of type 2 diabetes was assessed with restricted cubic splines. Splines with corresponding 95\% Cls from five imputation data sets are shown.

any diabetes-related complications seemed to be more pronounced in men (HR 1.50; $95 \% \mathrm{Cl} 1.23,1.83)$ compared with women $(1.19 ; 0.85,1.68)$ (Supplementary Fig. 4 and Supplementary Table 6). However, no statistically significant interaction between NT-proBNP levels and sex could be detected $(P=0.22)$. HRs for microvascular complications were $1.42(1.11,1.81)$ in men and $1.17(0.79,1.74)$ in women (Supplementary Fig. 4 and Supplementary Table 6). It was not possible to estimate the HRs for macrovascular complications in women due to insufficient case numbers in this subgroup $(n=15)$. Exclusion of participants with baseline $\mathrm{HbA}_{1 \mathrm{c}} \geq 6.5 \%$ did not substantially change the associations of NT-proBNP with any vascular complications of diabetes (data not shown).

\section{Prediction of Diabetes and Diabetes- Related Complications with NT-proBNP}

Regarding the predictive ability of NTproBNP for type 2 diabetes, the c-index $(95 \% \mathrm{Cl})$ was not changed upon addition of NT-proBNP to the German Diabetes Risk Score model: 0.853 (0.819, $0.884)$ vs. 0.852 (0.819, 0.883). Also, NT-proBNP alone was not able to discriminate between diabetes cases and noncases: c-index $0.546(0.502,0.590)$. For prediction of diabetes-related complications, however, the c-index improved from $0.681(95 \% \mathrm{Cl} 0.558,0.793)$ to 0.694 (95\% Cl 0.572, 0.804) upon addition of NT-proBNP to the model.

\section{CONCLUSIONS}

We observed that NT-proBNP was inversely associated with the risk of type 2 diabetes and that this association was more apparent in women in our study. In contrast to this inverse association, circulating levels of NT-proBNP were positively associated with vascular complications among individuals who developed type 2 diabetes during the follow-up, independent of major risk factors such as age, sex, BMI, sociodemographic and lifestyle factors, history of prevalent conditions, inflammatory and cardiometabolic biomarkers, and sex hormones.

The reported associations of high-normal NT-proBNP concentrations with lower diabetes risk in this study are in accordance with previously published findings from large epidemiological and Mendelian randomization studies $(5-8,28)$. Intriguingly, our data suggest that the contribution of this biomarker to the risk of type 2 diabetes is potentially modified by sex. Circulating NT-proBNP was observed to be affected by sex, with women showing higher levels of this peptide than men (16-18). This is an observation also made in our study. Previous studies observed a stimulatory effect of estrogens on the natriuretic peptide system (29-31), though these findings were inconsistent in humans (17). Estradiol appeared to regulate natriuretic peptide transcription and secretion and to augment NPR-A expression levels while stabilizing or reducing NPR-C transcription levels in mice $(29,30)$; and in humans, natriuretic peptides inversely correlated with androgen levels $(17,32)$ and were found to be lower in postmenopausal compared with premenopausal women $(16,17,31)$. Sex- and hormonedependent regulation of natriuretic peptides could at least partially account for the differences in cardiometabolic risk profiles between women and men by promoting adipose tissue dissemination from visceral to more favorable subcutaneous depots (33). Though we do not have measures of body composition in this study, hormone-dependent modulation of cardiometabolic risk through fat distribution by natriuretic peptides remains a possibility.

We could not detect any statistically significant linear associations of NT-proBNP with the risk of $\mathrm{Ml}$ or stroke in our study. Two systematic reviews showed a useful role of natriuretic peptides in cardiovascular risk assessment $(34,35)$. However, Mendelian randomization studies investigating the causal role of NT-proBNP in $\mathrm{MI}$ or stroke onset are lacking, and the systematic reviews included studies on high-risk populations and/or patients with stable CVD, and HF cases in the composite CVD outcome. It could be speculated that higher cardiac NT-proBNP levels in physiological conditions may be protective against type 2 diabetes and also against CVD $(3,36)$, acting against obesity and dysregulation of glucose/lipid metabolism, while higher levels in pathological conditions may represent a rescue mechanism of the cardiovascular system, which attempts to counteract the pathology by releasing the cardiac hormones. In line with previous studies (37), higher NT-proBNP levels were associated with a more favorable lipid profile, which are characterized by lower triglycerides and higher HDL-cholesterol concentrations in our study. This relation may further substantiate a positive role of NT-proBNP on cardiometabolic and cardiovascular risks in healthy physiology.

With regard to vascular complications of diabetes, several prospective studies in patients with diabetes reported 

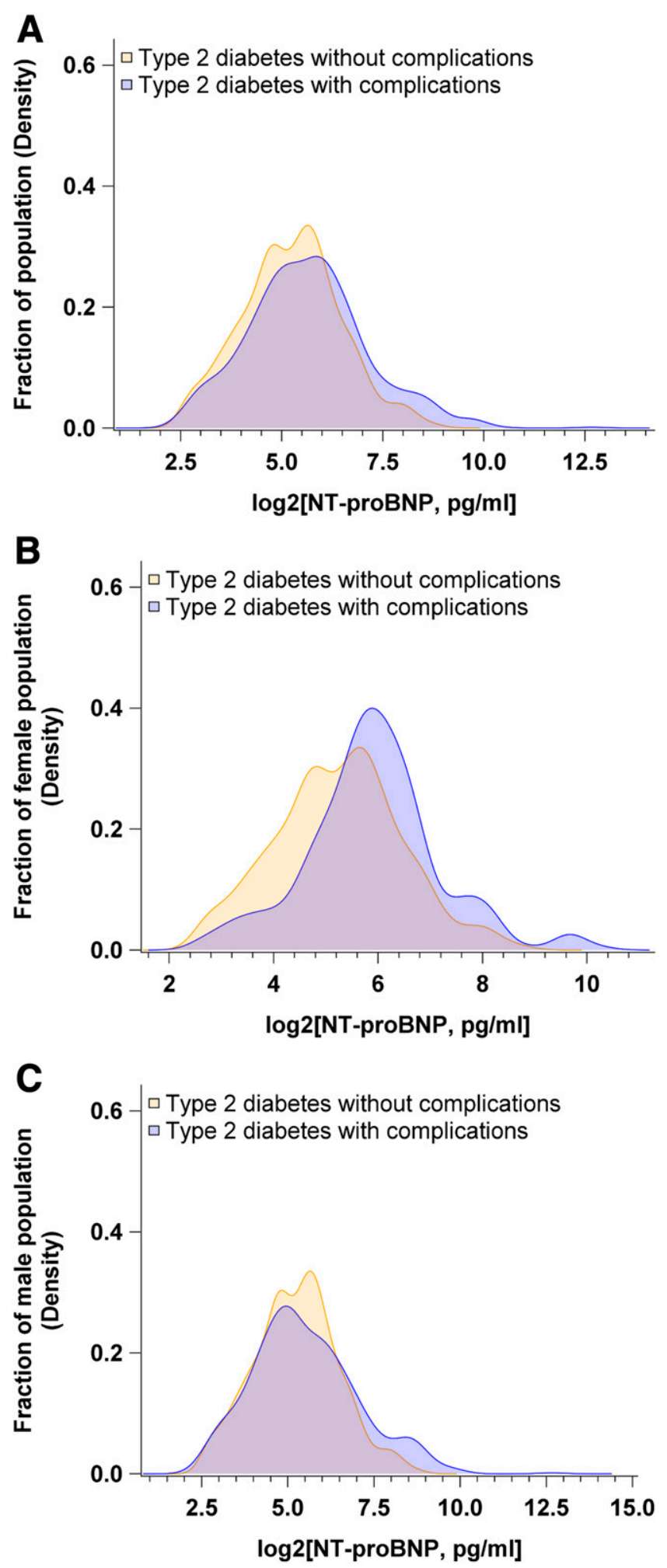

Figure 2-Density distribution of baseline NT-proBNP concentrations stratified by type 2 diabetes complications status and sex. A: Density distribution of baseline NT-proBNP concentrations in all participants with incident diabetes. $B$ : Density distribution of baseline NT-proBNP concentrations in women with incident diabetes. $C$ : Density distribution of baseline NT-proBNP concentrations in men with incident diabetes.

associations of higher NT-proBNP concentrations with an increased risk of CVD (11-15). However, prospective studies on microvascular complications are scarce. The only two prospective studies known to us are a case-cohort study nested within the Action in Diabetes and Vascular Disease: Preterax and Diamicron MR Controlled Evaluation (ADVANCE) trial (38) and a 9-month-long randomized controlled sulodexide macroalbuminuria (Sun-MACRO) trial (11). Both studies reported that NT-proBNP was associated with a risk of renal events, such as nephropathy (38) or loss of kidney function or end-stage renal disease (11). Also, in line with our findings, they showed that the discriminative performance of the models increased upon addition of NT-proBNP for prediction of nephropathy (38) or cardiovascular and renal events (11). However, these studies were limited as they did not report sex-specific associations, were of shorter duration, assessed NT-proBNP concentrations after individuals developed diabetes, and included patients with preexisting complications. Because NTproBNP levels in diseased populations might be altered due to a present or subclinical vascular, renal, and neurological impairment, reverse causation cannot be ruled out. In contrast, we measured NT-proBNP before diabetes onset and excluded participants with prevalent complications. Our data therefore provide new insights into the prospective contribution of this peptide to vascular and renal dysfunction that is assessed as microvascular events, in addition to its role as an established marker of cardiac impairment and stress. In individuals prone to develop diabetes, subclinical cardiac abnormalities as indicated by prediagnosis high-normal elevations in NT-proBNP may hint at physiologic stresses (extracellular volume expansion, left ventricular hypertrophy, silent myocardial ischemia, and reduced renal clearance). A strong association between asymptomatic cardiac impairment and kidney function decline exists even in the absence of clinically overt CVD $(39,40)$.

The major strength of our study is its population-based design in a low-risk setting and a use of both microvascular and macrovascular diabetes complications as an outcome in the same study. To our knowledge, this is the first study reporting on associations between baseline NT-proBNP and the risk of diabetesrelated vascular complications in the setting of an incident cohort with diabetes consisting of initially healthy individuals. Previous studies which reported the associations between peripheral NTproBNP and type 2 diabetes did not 

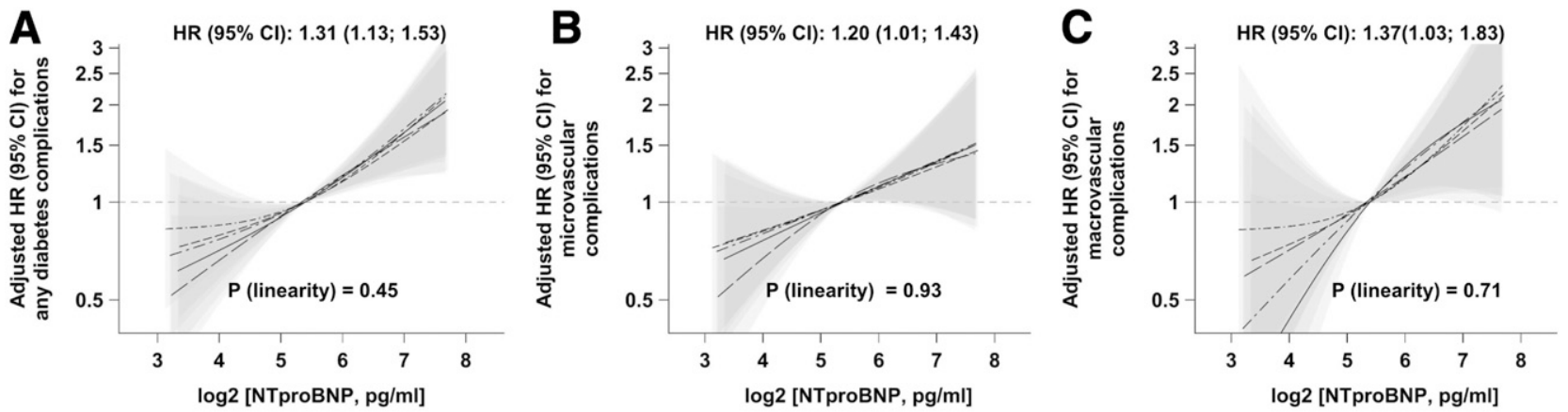

Figure 3-Associations of baseline NT-proBNP concentrations with risk of diabetes-related vascular complications in the patients with incident diabetes from the case-cohort, $n=545$. Adjusted HRs per doubling in NT-proBNP concentrations were assessed with Cox proportional hazards model (main model 2) and are shown with corresponding 95\% Cls the in the middle of each panel. Linearity of the relationship between NT-proBNP concentrations and risk of diabetic complications was assessed with restricted cubic splines. Splines with corresponding $95 \%$ Cls from five imputation data sets are shown. A: Associations with total vascular complications. B: Associations with microvascular complications. C: Associations with macrovascular complications. Microvascular complications were defined as new-onset retinopathy or blindness due to retinopathy, neuropathy or amputation due to neuropathy, nephropathy or kidney replacement therapy. Macrovascular complications were defined as newly diagnosed MI or stroke.

report the results separately for men and women $(6,8,11,38)$. Our finding that associations differ by sex should be confirmed in further studies. Further strengths include the rigorous validation of self-reported diagnoses, low drop-out rates, thorough adjustment for cardiometabolic risk factors, the large study sample, and long study follow-up.

Our study has some limitations. Diagnoses of nephropathy, neuropathy, and retinopathy were collected only in individuals which developed type 2 diabetes during follow-up. Moreover, the number of macrovascular complications was relatively low in our study. NT-proBNP measurements were done at recruitment, relatively long before the diabetes diagnosis, which was the new baseline for the analyses on complications. We tried to overcome these shortcomings by accounting for $\mathrm{HbA}_{1 \mathrm{c}}$ levels at recruitment and the duration between recruitment and diabetes onset in the statistical modeling. Furthermore, no repeated assessments of NT-proBNP concentrations were available in this study. It would be interesting to compare associations of pre- and postdiagnosis levels in terms of strength of association with complications. Study participants are ethnically almost exclusively of Caucasian ancestry; therefore, our findings might not be generalizable to other ethnicities and warrant an evaluation in other population groups. Finally, because of the observational character of this study, the observed associations cannot necessarily be considered causal.

In conclusion, peripheral NT-proBNP was inversely associated with the risk of type 2 diabetes in healthy individuals, particularly in women, whereas higher concentrations of this peptide related to higher risk of macrovascular and microvascular disease in people who develop diabetes. NT-proBNP may be a promising biomarker for detection of subclinical impairment in asymptomatic individuals at high risk for diabetes-related complications. Future studies should test the utility of NT-proBNP in risk stratification and tailoring therapeutic interventions for prevention of diabetes complications.

Acknowledgments. The authors thank the Human Study Centre of the German Institute of Human Nutrition Potsdam-Rehbrücke, namely, the trustee and the data hub for the processing, and the participants for the provision of the data, the biobank for the processing of the biological samples and the head of the Human Study Centre, Manuela Bergmann, for the contribution to the study design and leading the underlying processes of data generation.

Funding. The recruitment phase of the EPICPotsdam Study was supported by the Federal Ministry of Science, Germany (01 EA 9401) and the European Union (SOC 95201408 05F02). The follow-up of the EPIC-Potsdam Study was supported by German Cancer Aid (70-2488-Ha I) and the European Community (SOC 98200769 05F02). This work was furthermore supported by a grant from the German Ministry of Education and Research and the State of Brandenburg (DZD grant 82DZD00302).

Duality of Interest. No potential conflicts of interest relevant to this article were reported. Author Contributions. A.B. performed statis tical analyses and drafted the manuscript. A.B. and M.B.S. designed the study and interpreted the data. F.E., O.K., E.P., and J.W. contributed to statistical analyses, creation of graphs, and interpretation of data. A.F. and H.B. contributed to the acquisition and interpretation of data. C.W. contributed to study design and interpretation of results. All authors critically revised the manuscript. A.B. is the guarantor of this work and, as such, had full access to all the data in the study and takes responsibility for the integrity of the data and the accuracy of the data analysis.

\section{References}

1. Oremus $M$, McKelvie $R$, Don-Wauchope $A$, et al. A systematic review of BNP and NT-proBNP in the management of heart failure: overview and methods. Heart Fail Rev 2014;19:413-419 2. Sengenès $C$, Berlan M, De Glisezinski I, Lafontan M, Galitzky J. Natriuretic peptides: a new lipolytic pathway in human adipocytes. FASEB J 2000;14: 1345-1351

3. Gruden G, Landi A, Bruno G. Natriuretic peptides, heart, and adipose tissue: new findings and future developments for diabetes research. Diabetes Care 2014;37:2899-2908

4. Tsukamoto O, Fujita M, Kato M, et al. Natriuretic peptides enhance the production of adiponectin in human adipocytes and in patients with chronic heart failure. J Am Coll Cardiol 2009; 53:2070-2077

5. Pfister R, Sharp S, Luben R, et al. Mendelian randomization study of B-type natriuretic peptide and type 2 diabetes: evidence of causal association from population studies. PLoS Med 2011;8:e1001112

6. Everett BM, Cook NR, Chasman DI, et al. Prospective evaluation of B-type natriuretic peptide concentrations and the risk of type 2 diabetes in women. Clin Chem 2013;59:557-565 7. Lazo M, Young JH, Brancati FL, et al. NH2terminal pro-brain natriuretic peptide and risk of diabetes. Diabetes 2013;62:3189-3193

8. Brutsaert EF, Biggs ML, Delaney JA, et al. Longitudinal assessment of $\mathrm{N}$-terminal pro-Btype natriuretic peptide and risk of diabetes in older adults: the cardiovascular health study. Metabolism 2016;65:1489-1497

9. Gruden G, Barutta F, Chaturvedi N, et al. NH2terminal probrain natriuretic peptide is associated with diabetes complications in the EURODIAB Prospective Complications Study: the role of tumor necrosis factor- $\alpha$. Diabetes Care 2012; 35:1931-1936

10. Hamano K, Nakadaira I, Suzuki J, Gonai M. Nterminal fragment of probrain natriuretic peptide 
is associated with diabetes microvascular complications in type 2 diabetes. Vasc Health Risk Manag 2014;10:585-589

11. Bidadkosh A, Lambooy SPH, Heerspink HJ, et al. Predictive properties of biomarkers GDF15, NTproBNP, and hs-TnT for morbidity and mortality in patients with type 2 diabetes with nephropathy. Diabetes Care 2017;40:784-792

12. Gori M, Gupta DK, Claggett B, et al. Natriuretic peptide and high-sensitivity troponin for cardiovascular risk prediction in diabetes: the Atherosclerosis Risk in Communities (ARIC) study. Diabetes Care 2016;39:677-685

13. Winkler K, Wanner C, Drechsler C, Lilienthal J, März W, Krane V; German Diabetes and Dialysis Study Investigators. Change in N-terminal-pro-Btype-natriuretic-peptide and the risk of sudden death, stroke, myocardial infarction, and allcause mortality in diabetic dialysis patients [published correction appears in Eur Heart J 2008;29: 2445-2446]. Eur Heart J 2008;29:2092-2099

14. Scirica BM, Bhatt DL, Braunwald E, et al. Prognostic implications of biomarker assessments in patients with type 2 diabetes at high cardiovascular risk: a secondary analysis of a randomized clinical trial. JAMA Cardiol 2016;1:989-998 15. Hillis GS, Welsh P, Chalmers J, et al. The relative and combined ability of high-sensitivity cardiac troponin $\mathrm{T}$ and $\mathrm{N}$-terminal pro-B-type natriuretic peptide to predict cardiovascular events and death in patients with type 2 diabetes. Diabetes Care 2014;37:295-303

16. Lew J, Sanghavi M, Ayers CR, et al. Sex-based differences in cardiometabolic biomarkers. Circulation 2017;135:544-555

17. Lam CS, Cheng S, Choong K, et al. Influence of sex and hormone status on circulating natriuretic peptides. J Am Coll Cardiol 2011;58:618-626

18. Wirth J, Buijsse B, di Giuseppe R, et al. Relationship between $\mathrm{N}$-terminal pro-brain natriuretic peptide, obesity and the risk of heart failure in middle-aged German adults. PLoS One 2014;9:e113710

19. Boeing H, Korfmann A, Bergmann MM. Recruitment procedures of EPIC-Germany. European Investigation into Cancer and Nutrition. Ann Nutr Metab 1999;43:205-215

20. Boeing $H$, Wahrendorf J, Becker N. EPICGermany-A source for studies into diet and risk of chronic diseases. European Investigation into Cancer and Nutrition. Ann Nutr Metab 1999;43: 195-204

21. Schienkiewitz A, Schulze MB, Hoffmann K, Kroke $A$, Boeing $H$. Body mass index history and risk of type 2 diabetes: results from the European Prospective Investigation into Cancer and Nutrition (EPIC)-Potsdam Study. Am J Clin Nutr 2006; 84:427-433

22. Jacobs S, Kröger J, Floegel A, et al. Evaluation of various biomarkers as potential mediators of the association between coffee consumption and incident type 2 diabetes in the EPIC-Potsdam Study. Am J Clin Nutr 2014;100:891-900

23. Canales RA, Wilson AM, Pearce-Walker JI, Verhougstraete MP, Reynolds KA. Methods for handling left-censored data in quantitative microbial risk assessment. Appl Environ Microbiol 2018;84:e01203-18

24. Levey AS, Stevens LA, Schmid CH, et al.; CKD-EPI (Chronic Kidney Disease Epidemiology Collaboration). A new equation to estimate glomerular filtration rate. Ann Intern Med 2009;150: 604-612

25. Mühlenbruch K, Ludwig T, Jeppesen C, et al. Update of the German Diabetes Risk Score and external validation in the German MONICA/ KORA study. Diabetes Res Clin Pract 2014;104: 459-466

26. Mühlenbruch K, Paprott R, Joost HG, Boeing $\mathrm{H}$, Heidemann $\mathrm{C}$, Schulze MB. Derivation and external validation of a clinical version of the German Diabetes Risk Score (GDRS) including measures of HbA1c. BMJ Open Diabetes Res Care 2018;6:e000524

27. Jiang W, Wang J, Shen X, et al. Establishment and validation of a risk prediction model for early diabetic kidney disease based on a systematic review and meta-analysis of 20 cohorts. Diabetes Care 2020;43:925-933

28. Salomaa V, Havulinna A, Saarela O, et al. Thirtyone novel biomarkers as predictors for clinically incident diabetes. PLoS One 2010;5:e10100

29. Mahmoodzadeh S, Pham TH, Kuehne A, et al. $17 \beta$-Estradiol-induced interaction of $\mathrm{ER} \alpha$ with NPPA regulates gene expression in cardiomyocytes. Cardiovasc Res 2012;96:411-421

30. Belo NO, Sairam MR, Dos Reis AM. Impairment of the natriuretic peptide system in follitropin receptor knockout mice and reversal by estradiol: implications for obesity-associated hypertension in menopause. Endocrinology 2008; 149:1399-1406

31. Maffei S, Del Ry S, Prontera C, Clerico A Increase in circulating levels of cardiac natriuretic peptides after hormone replacement therapy in postmenopausal women. Clin Sci (Lond) 2001; 101:447-453

32. Bachmann KN, Huang S, Lee H, et al. Effect of testosterone on natriuretic peptide levels. J Am Coll Cardiol 2019;73:1288-1296

33. Neeland IJ, Winders BR, Ayers CR, et al. Higher natriuretic peptide levels associate with a favorable adipose tissue distribution profile. J Am Coll Cardiol 2013;62:752-760

34. Willeit $P$, Kaptoge $S$, Welsh $P$, et al.; Natriuretic Peptides Studies Collaboration. Natriuretic peptides and integrated risk assessment for cardiovascular disease: an individual-participantdata meta-analysis. Lancet Diabetes Endocrinol 2016;4:840-849

35. Di Angelantonio E, Chowdhury R, Sarwar N, et al. B-type natriuretic peptides and cardiovascular risk: systematic review and meta-analysis of 40 prospective studies. Circulation 2009;120: 2177-2187

36. Sarzani R, Spannella F, Giulietti F, Balietti P, Cocci G, Bordicchia M. Cardiac natriuretic peptides, hypertension and cardiovascular risk. High Blood Press Cardiovasc Prev 2017;24:115126

37. Spannella F, Giulietti F, Bordicchia M, Burnett JC Jr., Sarzani R. Association between cardiac natriuretic peptides and lipid profile: a systematic review and meta-analysis. Sci Rep 2019;9:19178

38. Welsh P, Woodward M, Hillis GS, et al. Do cardiac biomarkers NT-proBNP and hsTnT predict microvascular events in patients with type 2 diabetes? Results from the ADVANCE trial. Diabetes Care 2014;37:2202-2210

39. Agarwal R, Song RJ, Vasan RS, Xanthakis V. Left ventricular mass and incident chronic kidney disease. Hypertension 2020;75:702-706

40. Park M, Shlipak MG, Katz R, et al. Subclinical cardiac abnormalities and kidney function decline: the multi-ethnic study of atherosclerosis. Clin J Am Soc Nephrol 2012;7:1137-1144 